\title{
Implementation of transcatheter aortic valve replacement in California: Influence on aortic valve surgery
}

\author{
Steven Maximus, MD, ${ }^{\mathrm{a}}$ Jeffrey C. Milliken, MD, ${ }^{\mathrm{a}}$ Beate Danielsen, PhD, ${ }^{\mathrm{b}}$ Richard Shemin, MD, \\ Junaid Khan, MD, ${ }^{\mathrm{d}}$ and Joseph S. Carey, $\mathrm{MD}^{\mathrm{a}}$
}

\section{ABSTRACT}

Objective: Transcatheter aortic valve replacement (TAVR) procedures were introduced in 2011. Initially, procedures were limited to patients who were not surgical candidates, but subsequently high-risk surgical candidates were considered for TAVR. The influence on aortic valve surgery in California is unknown.

Methods: The California Office of Statewide Health Planning and Development hospitalized patient discharge database was queried for the years 2009 through 2014. isolated surgical aortic valve and aortic valve/coronary artery bypass graft (SAVR) and TAVR procedures were identified by International Classification of Diseases-9th revision clinical modification procedure codes. Seven TAVR programs were introduced in 2011, 12 in 2012, 3 in 2013, and 6 in 2014. SAVR procedure volumes were compared from the 2 years before institution with SAVR volumes during the year(s) after institution of the TAVR program in these 28 hospitals.

Results: Overall, surgical volumes increased during the first, second, and third years after implementation of TAVR procedures. Among 7 hospitals with 4-year programs, surgical volumes increased to a maximum of $15.5 \%$ during the third year, then began to decrease. The hospital performing the largest number of TAVR procedures showed a marked decrease in SAVR volume by the fourth year, suggesting a shift of SAVR candidates to TAVR. Among all hospitals with 4-year programs, TAVR exceeded SAVR procedures by the fourth year. In California overall, SAVR increased during 2011 through 2013, due primarily to increasing volume of isolated SAVR procedures. Statewide, isolated SAVR increased from a yearly average of 3111 procedures during 2009-2010 to 3592 $(+15.5 \%)$ in 2013 , then decreased slightly in 2014 . SAVR plus coronary artery bypass graft procedures decreased during the same time period.

Conclusions: After implementation of TAVR, hospital SAVR volumes increased moderately, then began to decrease by the fourth year, when TAVR volume exceeded SAVR. Surgical candidates may be identified during evaluation for TAVR, resulting in increased SAVR volume. Increasing SAVR volume may also be related to improved patient and provider awareness of aortic valve disease.

(J Thorac Cardiovasc Surg 2018;155:1447-56)

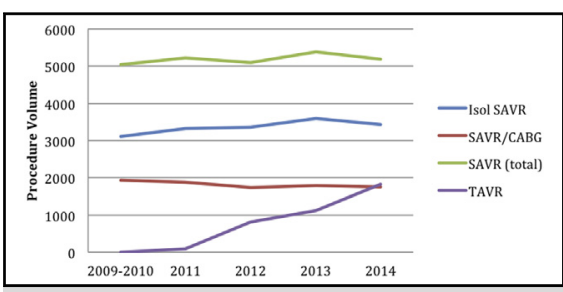

TAVR compared with SAVR over 4 years in California.

Central Message

Transcatheter aortic valve replacement procedure volume increased in California between 2011 and 2014

Perspective

The increase in transcatheter aortic valve (TAVR) procedures has had an unknown effect on surgical aortic valve replacement (SAVR). Our data show that, overall, SAVR volume remains relatively unchanged; however, by year 4 in 5 out of 7 hospitals performing TAVR, TAVR volume exceeded SAVR volume.

See Editorial Commentary page 1457

\footnotetext{
From the a Division of Cardiothoracic Surgery, Department of Surgery, University of California Irvine Medical Center, Orange, Calif; ' Health Information Solutions, Rocklin, Calif; ${ }^{\mathrm{c}}$ University of California Los Angeles Ronald Reagan Medical Center, Los Angeles, Calif; and ${ }^{\mathrm{d}}$ East Bay Cardiac Surgery Center, Oakland, Calif. Read at the 42nd Annual Meeting of The Western Thoracic Surgical Association, Waikoloa, Hawaii, June 22-25, 2016.

Received for publication Aug 15, 2016; revisions received July 14, 2017; accepted for publication July 21, 2017.

Address for reprints: Steven Maximus, MD, 333 The City Dr, Ste 1600, Orange, CA 92868 (E-mail: smaximus@uci.edu).

$0022-5223 / \$ 36.00$

Copyright (c) 2017 by The American Association for Thoracic Surgery

https://doi.org/10.1016/j.jtcvs.2017.07.092
}

Severe aortic disease is estimated to affect anywhere from $2 \%$ to $5 \%$ of people older than age 75 years, and untreated aortic stenosis is reported to have a mortality rate of $50 \%$ within 24 to 60 months. ${ }^{1}$ Traditionally, patients with severe

Scanning this QR code will take you to the article title page. 


\section{Abbreviations and Acronyms}

SAVR $=$ surgical aortic valve replacement

$\mathrm{TAVR}=$ transcatheter aortic valve replacement

aortic stenosis or with multiple comorbidities were not considered candidates for surgical aortic valve replacement (SAVR) due to their high surgical risk. ${ }^{2}$ Transcatheter aortic valve replacement (TAVR), was approved by the Food and Drug Administration in 2011, and was considered an alternative for patients who were believed to be too high risk for surgery. ${ }^{3}$ In patients who were not candidates for surgery, TAVR significantly reduced rates of death. ${ }^{3-5}$ Until recently, TAVR has been reserved for high-risk patients; however, recent studies have shown similar results in terms of mortality and morbidity in intermediate-risk patients. ${ }^{6}$ The influence of TAVR on surgical volumes in California is unknown, and we sought to examine the effect of TAVR on SAVR and the trend in terms of procedural volume in California hospitals.

\section{METHODS}

The California Office of Statewide Health Planning and Development Hospitalized Patient Discharge Database was queried for the years 2011 through 2014. The California Office of Statewide Health Planning and Development collects inpatient discharge data from all licensed inpatient hospitals in California. Each record in the dataset corresponds to an individual inpatient hospital discharge.

We queried the database from January 1, 2009, to December 31, 2014, for all patients undergoing a primary procedure of SAVR and TAVR. TAVR data became available in 2011. These patients were identified by International Classification of Diseases, 9th revision, clinical modification procedure codes. SAVR procedure volumes were compared from the 2 years prior and to SAVR volumes in the year(s) after institution of the TAVR program in these hospitals.

\section{RESULTS}

During 2011, a total of 5214 SAVR and 86 TAAVR procedures were performed in California hospitals (Table 1). Initially, only 7 of these hospitals performed TAVR during 2011, which increased to 28 hospitals by 2014 . The number of total SAVR procedures remained steady each year, with a slight decrease to 5185 in 2014 . The number of TAVR procedures increased each year, with 86 TAVR procedures

TABLE 1. Total number of aortic valve procedures performed in California, 2011-2014

\begin{tabular}{lrrrrr}
\hline & \multicolumn{5}{c}{ Annual procedure volume } \\
\cline { 2 - 6 } \multicolumn{1}{c}{ Procedure } & $\mathbf{2 0 0 9 - 2 0 1 0}$ & $\mathbf{2 0 1 1}$ & $\mathbf{2 0 1 2}$ & $\mathbf{2 0 1 3}$ & $\mathbf{2 0 1 4}$ \\
\hline Isolated SAVR & 3111 & 3333 & 3359 & 3592 & 3430 \\
SAVR + CABG & 1929 & 1881 & 1738 & 1789 & 1755 \\
SAVR (total) & 5040 & 5214 & 5097 & 5381 & 5185 \\
TAVR & 0 & 86 & 821 & 1128 & 1833 \\
\hline
\end{tabular}

SAVR, Surgical aortic valve replacement; $C A B G$, coronary artery bypass graft; $T A V R$, transcatheter aortic valve replacement.
TABLE 2. Number of surgical aortic valve replacement procedures in hospitals with 4-year transcatheter aortic valve replacement programs, by year*

\begin{tabular}{lrrrr}
\hline Hospital & $\mathbf{2 0 1 1}$ & $\mathbf{2 0 1 2}$ & $\mathbf{2 0 1 3}$ & $\mathbf{2 0 1 4}$ \\
\hline 1 & 222 & 143 & 156 & 99 \\
2 & 59 & 74 & 77 & 76 \\
3 & 19 & 29 & 34 & 23 \\
4 & 284 & 310 & 319 & 344 \\
\hline 5 & 77 & 102 & 119 & 91 \\
6 & 84 & 66 & 67 & 54 \\
7 & 85 & 81 & 99 & 124 \\
Total & 830 & 805 & 871 & 811 \\
\hline
\end{tabular}

*2009-2010 average for all hospitals was 754 surgical aortic valve replacement procedures.

performed in 7 different hospitals during 2011, which increased to 1833 TAVR procedures performed in 28 different hospitals by 2014 (Table 1). This represents a $0.6 \%$ decrease in SAVR volume compared with a $2131 \%$ increase in TAVR over the 4-year time period.

Overall, surgical volumes remained relatively unchanged in hospitals that implemented TAVR procedures during 2011 (Tables 2 and 3). Among the 7 hospitals with 4-year TAVR programs, 2-year average before TAVR implementation was 754 SAVR procedures. SAVR volumes increased to a maximum of 871 procedures during the third year ( $15.5 \%$ increase), then decreased to 811 ( $7.6 \%$ increase overall) by the fourth year. Tables 2 and 3 show individual hospital numbers over this time period in regard to hospitals that performed both SAVR and TAVR over 4 years. Hospital 1 performed the largest number of TAVR procedures (32 in 2011 and 377 in 2014 [1178\% increase]), and had a marked decrease in SAVR volume by the fourth year (from 222 to 99 [55.4\% decrease]). Among all hospitals with 4-year programs, TAVR exceeded SAVR procedures by the fourth year in 5 out of 7 hospitals. In California overall, SAVR increased during 2011 through 2013, due primarily to increasing the volume of isolated SAVR procedures. Overall procedure numbers from all hospitals from 2011-2014 for TAVR and SAVR are shown in Tables 4 and 5.

TABLE 3. Number of transcatheter aortic valve replacement (TAVR) procedures in hospitals with 4-year TAVR programs, by year

\begin{tabular}{lrrrr}
\hline Hospital & $\mathbf{2 0 1 1}$ & $\mathbf{2 0 1 2}$ & $\mathbf{2 0 1 3}$ & $\mathbf{2 0 1 4}$ \\
\hline 1 & 32 & 261 & 299 & 377 \\
2 & 5 & 26 & 41 & 65 \\
3 & 3 & 14 & 62 & 113 \\
4 & 4 & 22 & 33 & 67 \\
\hline 5 & 6 & 21 & 43 & 104 \\
6 & 24 & 105 & 120 & 157 \\
7 & 12 & 85 & 105 & 128 \\
Total & 86 & 534 & 702 & 1011 \\
\hline
\end{tabular}


TABLE 4. Number of surgical aortic valve replacement procedures in hospitals with transcatheter aortic valve replacement programs

\begin{tabular}{|c|c|c|c|c|}
\hline Hospital & 2011 & 2012 & 2013 & 2014 \\
\hline 1 & 222 & 143 & 156 & 99 \\
\hline 2 & 59 & 74 & 77 & 76 \\
\hline 3 & 19 & 29 & 34 & 23 \\
\hline 4 & 284 & 310 & 319 & 344 \\
\hline 5 & 77 & 102 & 119 & 91 \\
\hline 6 & 84 & 66 & 67 & 54 \\
\hline 7 & 85 & 81 & 99 & 124 \\
\hline 8 & - & 108 & 87 & 83 \\
\hline 9 & - & 71 & 53 & 60 \\
\hline 10 & - & 226 & 243 & 183 \\
\hline 11 & - & 35 & 36 & 27 \\
\hline 12 & - & 45 & 69 & 71 \\
\hline 13 & - & 66 & 65 & 58 \\
\hline 14 & - & 165 & 217 & 246 \\
\hline 15 & - & 84 & 90 & 83 \\
\hline 16 & - & 133 & 155 & 137 \\
\hline 17 & - & 64 & 68 & 56 \\
\hline 18 & - & 146 & 168 & 165 \\
\hline 19 & - & 45 & 59 & 50 \\
\hline 20 & - & - & 62 & 42 \\
\hline 21 & - & - & 45 & 43 \\
\hline 22 & - & - & 54 & 60 \\
\hline 23 & - & - & - & 49 \\
\hline 24 & - & - & - & 30 \\
\hline 25 & - & - & - & 33 \\
\hline 26 & - & - & - & 78 \\
\hline 27 & - & - & - & 152 \\
\hline 28 & - & - & - & 45 \\
\hline Total & 830 & 1993 & 2342 & 2562 \\
\hline
\end{tabular}

Table 6 shows the influence of TAVR on SAVR procedure volumes by year after implementation of TAVR programs. This is also illustrated in Figures 1 and 2. Overall surgical volumes increased after implementation of TAVR procedures. Among 7 hospitals with 4-year programs, surgical volumes increased to a maximum of $15.5 \%$ during the third year then began to decrease. In California overall, SAVR increased during 2011 through 2013, due primarily to increasing isolated SAVR procedures (Table 1). Statewide, isolated SAVR increased from a yearly average of 3111 procedures during 2009-2010 to 3592 (+15.5\%) during 2013, then decreased slightly in 2014. SAVR plus coronary artery bypass graft procedures decreased during the same time period (Table 1). Table 7 shows the mortality rate of the procedures. TAVR mortality decreased each year, with an $8.5 \%$ mortality rate in 2011 , which decreased to a mortality rate of $4.1 \%$ by 2014. Table 8 shows comorbidities of
TABLE 5. Number of transcatheter aortic valve replacement procedures by hospital, 2011-2014

\begin{tabular}{|c|c|c|c|c|}
\hline Hospital & 2011 & 2012 & 2013 & 2014 \\
\hline 1 & 32 & 261 & 299 & 377 \\
\hline 2 & 5 & 26 & 41 & 65 \\
\hline 3 & 3 & 14 & 62 & 113 \\
\hline 4 & 4 & 22 & 33 & 67 \\
\hline 5 & 6 & 21 & 43 & 104 \\
\hline 6 & 24 & 105 & 120 & 157 \\
\hline 7 & 12 & 85 & 105 & 128 \\
\hline 8 & - & 17 & 23 & 31 \\
\hline 9 & - & 17 & 18 & 49 \\
\hline 10 & - & 35 & 97 & 162 \\
\hline 11 & - & 6 & 6 & 14 \\
\hline 12 & - & 7 & 10 & 7 \\
\hline 13 & - & 3 & 6 & 18 \\
\hline 14 & - & 36 & 58 & 75 \\
\hline 15 & - & 11 & 23 & 39 \\
\hline 16 & - & 37 & 30 & 32 \\
\hline 17 & - & 22 & 29 & 67 \\
\hline 18 & - & 28 & 33 & 73 \\
\hline 19 & - & 32 & 41 & 68 \\
\hline 20 & - & - & 3 & 23 \\
\hline 21 & - & - & 2 & 39 \\
\hline 22 & - & - & 10 & 22 \\
\hline 23 & - & - & - & 35 \\
\hline 24 & - & - & - & 10 \\
\hline 25 & - & - & - & 8 \\
\hline 26 & - & - & - & 20 \\
\hline 27 & - & - & - & 23 \\
\hline 28 & - & - & - & 7 \\
\hline Total & 86 & 821 & 1128 & 1833 \\
\hline
\end{tabular}

patients undergoing TAVR. Overall, comorbidities were similar over the time period for most categories; however, $56 \%$ of patients undergoing TAVR during 2011 required preoperative intra-aortic balloon pump, which decreased to $11 \%$ of patients in 2014 (Table 9 shows that the majority of patients undergoing TAVR were those in the age group over 80).

\section{DISCUSSION}

We evaluated the influence of TAVR on SAVR volume by analyzing the number of procedures of TAVR and SAVR in the State of California. The data collected show that the number of TAVR procedures increased from 86 procedures to 1833 procedures over the time period 2011-2014. The number of hospitals with TAVR programs went from 8 in 2011 to 28 in 2014. The overall influence of TAVR over 
TABLE 6. Surgical aortic valve replacement (SAVR) and SAVR + coronary artery bypass graft (CABG) procedure volumes by year(s) after transcatheter aortic valve replacement (TAVR) program implementation

\begin{tabular}{|c|c|c|c|c|c|c|}
\hline \multirow[b]{2}{*}{ Hospitals (n) } & \multicolumn{5}{|c|}{ SAVR and SAVR + CABG } & \multirow[b]{2}{*}{ TAVR } \\
\hline & Previous 2-y average & Year 1 & Year 2 & Year 3 & Year 4 & \\
\hline 28 & 2497.5 & $2566(+2.7 \%)$ & & & & 455 (y 1) \\
\hline 22 & 2084.5 & $2179(+4.6 \%)$ & $2260(+8.4 \%)$ & & & 994 (y 2) \\
\hline 19 & 1953.5 & $2018(+3.3 \%)$ & $2115(+8.3 \%)$ & $2090(+7.0 \%)$ & & 1338 (у 3) \\
\hline 7 & 754 & $830(+10.1 \%)$ & $805(+6.8 \%)$ & $871(+15.5 \%)$ & $811(+7.6 \%)$ & $1011(\mathrm{y} 4)$ \\
\hline
\end{tabular}

SAVR, Surgical aortic valve replacement; $C A B G$, coronary artery bypass graft; $T A V R$, transcatheter aortic valve replacement.

this time period on SAVR seems to be minimal, because the number of SAVRs was 5214 in 2011 compared with 5185 in 2014 , which represents a $0.6 \%$ change (Table 1 ).

Tables 2 and 3 show individual hospital numbers over this time period in regard to 4-year programs with SAVR and TAVR. As shown in these Tables, the hospital that performed the most TAVR procedures in 2011-2014 showed a marked decrease in SAVR procedures by the fourth year, suggesting a shift of SAVR candidates to TAVR as institutions gain more experience with the procedure. This finding may indicate that lower-risk patients are now undergoing TAVR, given the change in SAVR and TAVR volumes seen in 4-year programs. Although most of the comorbidities in patients undergoing TAVR were relatively constant during this time period, the percentage of patients requiring intra-aortic balloon pump decreased from 56\% in 2011 to $11 \%$ in 2014 . This significant reduction may be partly explained by the exponential increase in TAVR volume; however, this indicates that patients with lower risk profiles are undergoing TAVR.

Reinohl and colleagues ${ }^{7}$ demonstrated similar findings to our study in Germany, where they found that TAVR procedures increased from 144 in 2007 to 9147 by 2013, with a moderate decrease in SAVR from 8622 to 7048 over the same time period. They found that TAVR patients tended to be older (age $>75$ years) had more comorbidities, and were at higher procedural risk of periprocedural and postprocedure morbidity and mortality. They attributed the increase in TAVR without large decrease in SAVR procedures to the fact that the patients undergoing TAVR

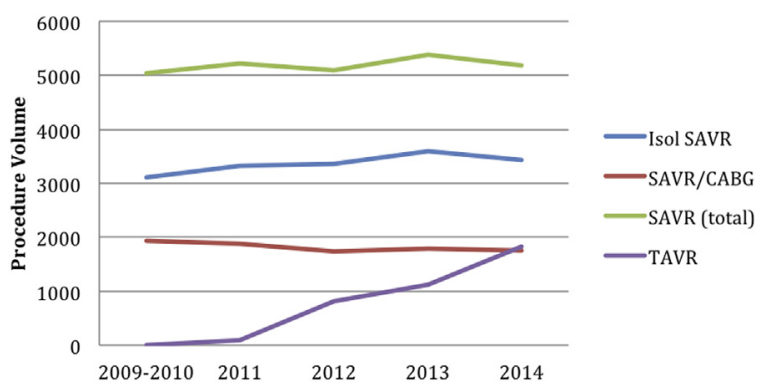

FIGURE 1. California annual surgical aortic valve replacement $(S A V R)$ and transcatheter aortic valve replacement $(T A V R)$ volume after TAVR was initiated. $C A B G$, Coronary artery bypass graft. would have been unlikely to undergo surgery due to their age and high-risk profile. ${ }^{7}$ Hannan and colleagues ${ }^{8}$ analyzed the New York State Cardiac Surgery reporting system over 2 years and found that the total number of aortic valve replacements increased from 2291 in 2011 to 2899 in 2012 ( $27 \%$ increase). The volume of patients undergoing SAVR increased from 1994 to $2135(7.1 \%)$ and the volume of TAVR patients increased from 297 to $764(157 \%)$ over the 1-year time period. The percentage of SAVR patients who were higher risk (Society of Thoracic Surgeons score, $8 \%$ ) decreased from $27 \%$ to $23 \%$, and the percentage of TAVR patients who were high risk decreased from $83 \%$ to $76 \%$. There was no significant difference in 1-year mortality between the 2 groups over the 1-year time interval analyzed. ${ }^{8}$ The authors also believed that patients with severe aortic stenosis were not considered surgical candidates due to their high perioperative risk; however, given the shift in the volume of TAVR procedures with moderate decreases in SAVR, TAVR had become an option for these patients and that this lead to the changes in procedural volume.

Multiple studies have shown short-term decreased mortality with similar outcomes in high-risk patients undergoing TAVR, which may explain the robust increase in the amount of TAVR procedures performed in California without a corresponding decrease in SAVR volume. Smith and colleagues ${ }^{9}$ showed no difference in mortality at 1 year in a study of 348 TAVR and 351 SAVR patients who were randomized at 25 different centers. They found that the mortality rate was $24.2 \%$ in the TAVR group compared with $26.8 \%$ in the SAVR group, which was not

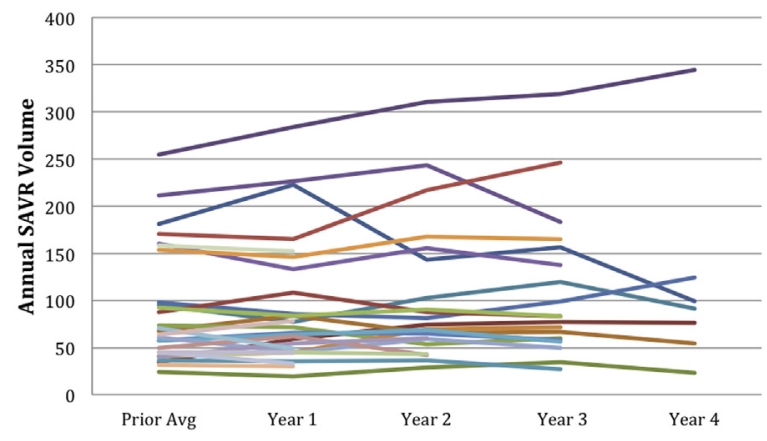

FIGURE 2. Hospital annual surgical aortic valve replacement (SAVR) volume after transcatheter aortic valve replacement (TAVR) was initiated. 
TABLE 7. Mortality of surgical aortic valve replacement (SAVR) + coronary artery bypass graft (CABG), SAVR, and transcatheter aortic valve replacement (TAVR) procedures

\begin{tabular}{|c|c|c|c|c|c|c|}
\hline \multirow[b]{2}{*}{ Year } & \multicolumn{2}{|c|}{ SAVR + CABG } & \multicolumn{2}{|c|}{ SAVR } & \multicolumn{2}{|c|}{ TAVR } \\
\hline & $\mathbf{N}$ & Mortality (\%) & $\mathbf{N}$ & Mortality (\%) & $\mathbf{N}$ & Mortality (\%) \\
\hline 2009 & 1930 & 5.1 & 3141 & 3.4 & & \\
\hline 2010 & 1829 & 5.7 & 3136 & 3 & & \\
\hline 2011 & 1660 & 6.3 & 3260 & 2.6 & 86 & 8.5 \\
\hline 2012 & 1738 & 6.2 & 3359 & 2.8 & 821 & 6.5 \\
\hline 2013 & 1789 & 5.9 & 3592 & 2.7 & 1128 & 4.5 \\
\hline 2014 & 1755 & 6 & 3430 & 2.4 & 1833 & 4.1 \\
\hline
\end{tabular}

$S A V R$, Surgical aortic valve replacement; $C A B G$, coronary artery bypass graft; $T A V R$, transcatheter aortic valve replacement.

statistically different. ${ }^{9}$ Kodali and colleagues ${ }^{10}$ showed similar results in a 2-year period: a $34 \%$ TAVR mortality rate compared with a $35 \%$ SAVR mortality rate. Adams and colleagues ${ }^{11}$ showed a 1 -year mortality rate of $14.2 \%$ in TAVR patients versus $19.1 \%$ in SAVR patients.

Studies over longer time periods have also shown efficacy of TAVR in high-risk patient populations. In a prospective, multicenter randomized trial of 750 high-risk patients with severe aortic stenosis, TAVR patients were compared with SAVR patients, and TAVR patients had less all-cause mortality $(32.9 \%$ vs $39.1 \%[P=.068])$, all stroke $(12.6 \%$ vs $19.0 \%[P=.034])$, and major adverse cardiovascular or cerebrovascular events $(40.2 \%$ vs $47.9 \%$, $[P=.025])$. At 3 years, aortic valve hemodynamic parameters were better with TAVR patients (mean aortic valve gradient, $7.62-3.57 \mathrm{~mm} \mathrm{Hg}$ vs11.40-6.81 $\mathrm{mm} \mathrm{Hg}$ in SAVR $[P<.001])$, although moderate or severe residual aortic regurgitation was higher in TAVR patients $(6.8 \%$ vs $0.0 \%$ in SAVR $[P<.001])$. There was no clinical evidence of valve thrombosis in either group. ${ }^{12}$

The Italian Observational Study of Effectiveness of SAVR-TAVI Procedures for Severe Aortic Stenosis Treatment (OBSERVANT) Study included 5707 SAVR patients and 1911 TAVR patients. A matched population of intermediate-risk patients was created that had a total of 650 patients for each group. At 1 year, the mortality rate was $13.6 \%$ in the SAVR group and $13.8 \%$ in the TAVR group $(P=.936)$. Major cardiovascular and cerebrovascular events were similar: $17.6 \%$ and $18.2 \%$, respectively $(P=.831)$. The authors did caution that more long-term randomized trials needed to be done, but short-term results did not show a statistical difference in mortality, cerebrovascular events, and rehospitalization. ${ }^{13}$

A single-institution study done at the University of Alabama analyzed the influence of TAVR on SAVR volumes. The authors reported an overall $12 \%$ increase in SAVR

TABLE 8. Comorbidities of patients undergoing transcatheter aortic valve replacement

\begin{tabular}{|c|c|c|c|c|c|c|c|c|}
\hline \multirow[b]{2}{*}{ Comorbidity } & \multicolumn{2}{|c|}{2011} & \multicolumn{2}{|c|}{2012} & \multicolumn{2}{|c|}{2013} & \multicolumn{2}{|c|}{2014} \\
\hline & $\overline{\mathbf{n}}$ & $\%$ & $\mathbf{n}$ & $\%$ & $\mathbf{n}$ & $\%$ & $\mathbf{n}$ & $\%$ \\
\hline Dialysis & 1 & 1.09 & 7 & 0.85 & 18 & 1.60 & 32 & 1.70 \\
\hline Status of procedure urgent & 0 & 0.00 & 2 & 0.24 & 3 & 0.27 & 7 & 0.37 \\
\hline Atrial fibrillation and flutter & 31 & 33.70 & 316 & 38.49 & 401 & 35.55 & 709 & 37.73 \\
\hline Cardiogenic shock & 1 & 1.09 & 7 & 0.85 & 10 & 0.89 & 16 & 0.85 \\
\hline Peripheral vascular disease & 28 & 30.43 & 186 & 22.66 & 354 & 31.38 & 591 & 31.45 \\
\hline Chronic lung disease & 25 & 27.17 & 181 & 22.05 & 270 & 23.94 & 381 & 20.28 \\
\hline Cerebrovascular disease & 7 & 7.61 & 81 & 9.87 & 94 & 8.33 & 197 & 10.48 \\
\hline Diabetes & 32 & 34.78 & 252 & 30.69 & 356 & 31.56 & 654 & 34.81 \\
\hline Renal failure & 41 & 44.57 & 322 & 39.22 & 428 & 37.94 & 751 & 39.97 \\
\hline Cardiomyopathy & 7 & 7.61 & 57 & 6.94 & 95 & 8.42 & 166 & 8.83 \\
\hline Heart failure & 68 & 73.91 & 598 & 72.84 & 772 & 68.44 & 1289 & 68.60 \\
\hline Infectious endocarditis & 0 & 0.00 & 4 & 0.49 & 1 & 0.09 & 2 & 0.11 \\
\hline Preoperative intra-aortic balloon pump & 52 & 56.52 & 269 & 32.76 & 183 & 16.22 & 212 & 11.28 \\
\hline Acute myocardial infarction present at scheduled admission & 0 & 0.00 & 8 & 0.97 & 11 & 0.98 & 22 & 1.17 \\
\hline Acute myocardial infarction present at unscheduled admission & 2 & 2.17 & 16 & 1.95 & 14 & 1.24 & 29 & 1.54 \\
\hline Prior coronary artery bypass graft or valve surgery & 15 & 16.30 & 168 & 20.46 & 270 & 23.94 & 413 & 21.98 \\
\hline
\end{tabular}


TABLE 9. Age groups of patients undergoing transcatheter aortic valve replacement procedures

\begin{tabular}{|c|c|c|c|c|c|c|c|c|}
\hline \multirow[b]{2}{*}{ Age at admission (y) } & \multicolumn{2}{|c|}{2011} & \multicolumn{2}{|c|}{2012} & \multicolumn{2}{|c|}{2013} & \multicolumn{2}{|c|}{2014} \\
\hline & $\bar{n}$ & $\%$ & $\mathbf{n}$ & $\%$ & $\mathbf{n}$ & $\%$ & $\mathbf{n}$ & $\%$ \\
\hline $18-50$ & 0 & 0.00 & 8 & 0.97 & 16 & 1.42 & 17 & 0.90 \\
\hline $51-55$ & 0 & 0.00 & 5 & 0.61 & 8 & 0.71 & 15 & 0.80 \\
\hline $56-60$ & 0 & 0.00 & 13 & 1.58 & 14 & 1.24 & 25 & 1.33 \\
\hline $61-65$ & 2 & 2.17 & 16 & 1.95 & 32 & 2.84 & 46 & 2.45 \\
\hline $66-70$ & 6 & 6.52 & 36 & 4.38 & 61 & 5.41 & 94 & 5.00 \\
\hline $71-75$ & 3 & 3.26 & 45 & 5.48 & 83 & 7.36 & 140 & 7.45 \\
\hline $76-80$ & 9 & 9.78 & 96 & 11.69 & 150 & 13.30 & 224 & 11.92 \\
\hline$<80$ & 72 & 78.26 & 602 & 73.33 & 764 & 67.73 & 1318 & 70.14 \\
\hline
\end{tabular}

volume 2.5 years after establishing a TAVR program. They reported an increase of $266 \%$ in TAVR volume. Overall volume increase of aortic valve replacements in both surgical and nonsurgical groups was attributed to the inclusion of high-risk patients who may not have been evaluated before TAVR program initiation. ${ }^{14}$

Brennan and colleagues ${ }^{15}$ also showed similar results, finding that overall SAVR increased by $16 \%$ in nonTAVR hospitals compared with $22 \%$ increases in hospitals with TAVR programs from 2008 to 2013. Other studies have also shown increases in SAVR volume with initiation of TAVR programs. A single-center study in the United Kingdom ${ }^{16}$ reported a $37 \%$ increase in SAVR over 2 years after implementation of a TAVR program, and another single-center study in the United States ${ }^{17}$ showed a $10 \%$ reduction in the rate of unoperated aortic stenosis. The data in our study shows a decrease in SAVR volume at the 4-year mark, as well as TAVR volume exceeding SAVR volume in 5 out of 7 hospitals with 4-year TAVR programs. Although the influence on SAVR may be subtle in terms of overall volume, TAVR has a significant influence on SAVR volume in individual centers.

Given the increase in overall aortic valve replacement procedures, and the concomitant rise in SAVR procedures, one can infer that patients who were not considered or referred for aortic valve replacements are now being referred for evaluation and treatment. The best treatment modality for each individual is still in question, and more data are needed to answer this question. In high-risk surgical patients, TAVR has shown a benefit compared with medical therapy. In low-risk patients, the data are not as clear, and although TAVR patients may have comparable early survival, low-risk SAVR patients may have improved long-term survival and fewer complications. ${ }^{18}$ The long-term durability of TAVR valves has also been brought into question because recent data have shown that a significant number of TAVR valves degenerate. ${ }^{19}$ Kaplan-Meier estimates done by the investigators ${ }^{19}$ estimated an 8-year structural valve degeneration rate of $50 \%$. More long-term information is needed before expanding TAVR to low-risk patients, and the results of long-term data may influence the role of TAVR in intermediate-risk patients.

Limitations of this study are that the data from this project come from an administrative data set that is dependent on administrative and billing coding. Administrative databases are designed for their own individual purposes (eg, administrative claims) and not designed for clinician inference. ${ }^{20} \mathrm{It}$ is also unknown how many TAVR procedures were part of a research protocol. Both commercial and trial cases would have been possible during the time period of this study; however, due to the fact that these data come from an administrative dataset, it is not possible to ascertain whether or not the procedure was part of a research protocol or trial case without access to the original patient information. Appropriate risk adjustment outcomes in patients undergoing TAVR at 4-year programs would be helpful to determine whether intermediate- and low-risk patients are indeed undergoing TAVR as opposed to SAVR at these institutions.

\section{CONCLUSIONS}

Overall, the increase in volume of aortic valve replacement procedures in both surgical and nonsurgical groups may be due to the inclusion of high-risk patients who may not have been evaluated before TAVR program initiation. As we have shown in our study, the TAVR volume exceeded SAVR volume in 5 out of 7 hospitals with 4-year TAVR programs. The trends in this study show that although there is an initial increase in the volume of SAVR procedures in institutions that initiate TAVR programs, the decrease in volume of SAVR at 4-year programs may indicate that patients who present intermediate and low risk are being shifted toward TAVR.

In high-risk surgical patients, TAVR has shown a benefit compared with medical therapy. In low-risk patients, the data are not as clear. Recent studies have questioned the benefit of TAVR in low-risk surgical populations, and other recent studies have indicated a significant long-term valve degeneration rate. 
As TAVR procedures continue to increase in volume, long-term investigation of the viability of TAVR is warranted. The volume should continue to be tracked, as should long-term outcomes of high-, intermediate-, and low-risk patients.

\section{Conflict of Interest Statement}

Authors have nothing to disclose with regard to commercial support.

\section{References}

1. Yarbrough W, Ikonomidis J. Aortic valve replacement. In: Yuh D, Varicella L, Yang S, Doty J, eds. Textbook of Cardiothoracic Surgery. 2nd ed. New York: McGraw Hill; 2014:501-24.

2. Supino PG, Borer JS, Preibisz J, Bornstein A. The epidemiology of valvular heart disease: a growing public health problem. Heart Fail Clin. 2006;2:379-93.

3. Leon MB, Smith CR, Mack M, Moses JW, Svensson LG, Tuzcu EM, et al. Transcatheter aortic-valve implantation for aortic stenosis in patients who cannot undergo surgery. N Engl J Med. 2010;363:1597-607.

4. Holmes DR Jr, Brennan JM, Rumsfeld JS, Dai D, O'Brien SM, Vemulapalli S, et al; for the STS TVT Registry. Clinical outcomes at 1 year following transcatheter aortic valve replacement. JAMA. 2015;313:1019-28.

5. Reardon MJ, Adams DH, Kleiman NS, Yakubov SJ, Coselli JS, Deeb GM, et al. 2-Year outcomes in patients undergoing surgical or self-expanding transcatheter aortic valve replacement. J Am Coll Cardiol. 2015;66:113-21.

6. Leon MB, Smith CR, Mack MJ, Makkar RR, Svenson LG, Kodali SK, et al. Transcatheter or surgical aortic-valve replacement in intermediate-risk patients. N Engl J Med. 2016;374:1609-20.

7. Reinohl J, Kaier K, Reinecke H, Schoor C, Frankenstin L, Vach W, et al. Effect of availability of transcatheter aortic-valve replacement on clinical practice. $N$ Engl J Med. 2015;375:2438-47.

8. Hannan EL, Samadashvili Z, Stamato NJ, Lahey SJ, Wechsler A, Jordan D, et al. Utilization and 1 year mortality for transcatheter aortic valve replacement and surgical aortic valve replacement in New York patients with aortic stenosis: 2011-2012. JACC Cardiovasc Interv. 2016;9:578-85.

9. Smith CR, Leon MB, Mack MJ, Miller C, Moses JW, Svenson LG, et al. Transcatheter versus surgical aortic-valve replacement in high-risk patients. $N$ Engl J Med. 2011;364:2187-98.

10. Kodali S, Pibarot P, Douglas PS, Williams M, Xu K, Thourani V, et al. Paravalvular regurgitation after transcatheter aortic valve replacement with the Edwards Sapien valve in the PARTNER trial: characterizing patients and impact on outcomes. Eur Heart J. 2015;36:449-56.

11. Adams DH, Popma JJ, Reardon MJ, Patel HJ, Grossman PM, Yakubov SJ, et al. Transcatheter aortic-valve replacement with a self-expanding prosthesis. $N$ Engl J Med. 2014:370:1790-8.

12. Deeb GM, Reardon MJ, Chetcuti SC, Patel HJ, Grossmark PM, Yakubor SJ, et al. 3 -year outcomes in high risk patients who underwent surgical or transcatheter aortic valve replacement. J Am Coll Cardiol. 2016;67:2565-74.

13. Tamburino C, Barbanti M, D'Errigo P, Ranucci M, Onorati F, Santini F, et al. 1Year outcomes after transfemoral transcatheter or surgical aortic valve replacement: results from the Italian OBSERVANT study. J Am Coll Cardiol. 2015; 66:804-12.

14. Davies JE, McAlexander WW, Sasse MF, Leesar MA, Melby SJ, Singh SP, et al Impact of transcatheter aortic valve replacement on surgical volumes and outcomes in a tertiary academic cardiac surgical practice. J Am Coll Surg. 2016; 222:645-55.

15. Brennan JM, Holmes DR, Sherwood MW, Edwards FH, Carroll JD, Grover FL, et al. The association of transcatheter aortic valve replacement availability and hospital aortic valve replacement volume and mortality in the United States. Ann Thorac Surg. 2014;98:2016-22.

16. Grant SW, Devbhandari MP, Grayson AD, Dimarakas I, Kadir I, Saravanan DM, et al. What is the impact of providing a transcatheter aortic valve implantation service on conventional aortic valve surgical activity: patient risk factors and outcomes in the first 2 years. Heart. 2010;96:1633-7.

17. Malaisrie SC, Tuday E, Lapin B, Wang E, Lee R, McGee EC, et al. Transcatheter aortic valve implantation decreases the rate of unoperated aortic stenosis. Eur J Cardiothorac Surg. 2011;40:43-8.
18. Rosato S, Santini F, Barbanti M, Biancari F, D'Errigo P, Onorati F, et al. Transcatheter aortic valve implantation compared with surgical aortic valve replacement in low-risk patients. Circ Cardiovasc Interv. 2016;9:e003326.

19. Dvir D. First look at long-term durability of transcatheter heart valves: assess ment of valve function up to 10 years after implantation. Presented at: EuroPCI; May 19, 2016; Paris, France.

20. Geraci JM, Johnson ML, Gordon HS, Petersen NJ, Shroyer AL, Grover FL, et al Mortality after cardiac bypass surgery: prediction from administrative versus clinical data. Med Care. 2005;43:149-58.

Key Words: transcatheter aortic vavle replament, surgical aortic valve replacement, TAVR, SAVR

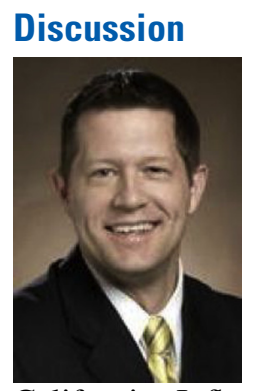

Dr Michael Weyant. So we'll now begin Scientific Session II. There are 4 presentations during this session, and I think it's important to note that all 4 of the presenters are candidates for the Samson Award. First presentation is "Implementation of Transcatheter Aortic Valve Replacement in California: Influence on Aortic Valve Surgery." This will be presented by Steven Maximus from UC Irvine.

Dr Steven Maximus (Orange, Calif). Good morning. I'd like to thank the Association for the opportunity to present our project. Traditionally, patients with severe aortic stenosis with multiple comorbidities were not considered candidates for surgical aortic valve replacement (SAVR) due to their high surgical risk. Transcatheter aortic valve replacement (TAVR) was introduced in 2011 and was considered an alternative for patients who were believed to be too high-risk for surgery. In patients who are not candidates for surgery, TAVR has significantly reduced rates of death compared with medical management. Until recently, TAVR has been reserved for high-risk patients; however, recent studies have shown similar results in terms of mortality and morbidity in intermediate-risk patients. The influence of TAVR on surgical volumes in California is unknown, and we sought to examine the effect of TAVR on SAVR and the trends in terms of procedure volume in California hospitals. We used the California Office of Statewide Health Planning and Development Hospitalized Patient Discharge Database and we curated this from the years 2011 to 2014. The California Office of Statewide Health Planning and Development collects inpatient discharge data form all licensed inpatient hospitals in California. The year 2011 was the first year TAVR data were available and we compared SAVR procedure volumes from the 2 years before SAVR volumes in the years after institution of the TAVR program in these hospitals. So these are the results. In 2011, there were a total of 5214 SAVRs. During that same year, there were 86 TAVRs. Initially, only 7 hospitals in California performed TAVRs in 2011, but by 2014, this increased to 28 hospitals. Statewide, isolated 
SAVR increased from a yearly average of 3111 procedures in 2009 and 2010 to 3592 in 2013, which represents a 15\% increase, then decreased slightly in 2014. The SAVR plus coronary artery bypass graft procedures remained about the same during this time period. The total number of SAVR procedures remains steady each year with a slight decrease to 5185 in 2014. The number of TAVRs increased each year: 86 TAVRs in 7 different hospitals in 2011, which increased to 1833 procedures in 20 different hospitals by 2014. This represents a $0.6 \%$ decrease in surgical volume compared with more than a $2000 \%$ increase in TAVR procedures over the same 4-year time period. And again, this is a chart showing the same thing, basically the number of TAVR procedures increased each year as you can see from 2011 to 2014. You've got 7 different hospitals in 2011 to 20 different hospitals in 2014. More than 1800 were performed in 2014. Again, that represents more than a $2000 \%$ increase.

Breaking this down in terms of hospitals that have actually done TAVRs for the past 4 years, from 2011 to 2014, the hospitals correspond to the same in terms of hospital 1 is the same on both charts and so forth. The first table shows the number of SAVR procedures in hospitals with 4-year TAVR programs. As you can see, hospital 1 performed the largest number of TAVR procedures during 2014. They went from 32 in 2011 to 377 in 2014-that's a more than $1000 \%$ increase. They also had a marked decrease in their SAVR volume by the fourth year. They went from 222 in 2011 to 99 in 2014, that's a 55.4\% decrease. Among all the hospitals with 4-year programs, which are all listed here, TAVR exceeded SAVR procedures in the fourth year in 5 out of 7 hospitals. As shown in the table that I showed before, the surgical volume for the state as a whole, in other words, the overall influence of TAVR on SAVRs, seemed to be minimal because the number of SAVRs was 5214 in 2011, compared with 5185 in 2014 - that's about a $0.6 \%$ change - so barely a minimal change. However, when you analyze these numbers by individual hospitals, you can see these different trends. Again, the hospital that performed the most TAVRs showed a marked decrease in SAVR procedures by the fourth year. This suggests a shift of SAVR candidates to TAVR as institutions gain more experience with the procedure. This finding may indicate that lowerrisk patients are now undergoing TAVR given the change in the SAVR and TAVR volumes seen in 4-year programs.

These are mortality numbers. The surgical aortic valve plus coronary artery bypass graft mortality was between $5.7 \%$ and $6.3 \%$ varied per year, overall pretty steady. The TAVR mortality went from $8.5 \%$ in 2011 down to $4.1 \%$ in 2014 , obviously the end number changed greatly here. There's a couple of reasons why the mortality numbers probably went down: the practitioner's experience with the procedure improved, but also the decrease in isolated surgical aortic valve mortality has been described in other studies as well, and this increase in surgical aortic valve volume combined with decreased mortality is known as the halo effect and it's described in other studies. Basically, the idea is that you have sicker patients who are being evaluated for TAVR who actually end up becoming surgical candidates, so henceforth, the increase in surgical volume. This is believed to be due to increased referral patterns for aortic valve patients and high-risk surgical patients undergoing TAVR.

Obviously, these data come from an administrative dataset and data are only as good as the coding being done, you need the ICD codes for diagnosis and now the ICD-10 codes because we've changed to that. We don't have data on patient demographic characteristics, comorbidities, or risk factors, and we have limited outcome data, really just mortality at this point. Analyzing the trend of the comorbidity and risk profile of patients undergoing TAVR at 4-year institutions would have been helpful to determine whether intermediate and low-risk patients are indeed undergoing TAVR as opposed to SAVR at these institutions.

In conclusion, traditionally, patients with severe aortic stenosis or with multiple medical comorbidities weren't considered candidates for SAVR due to their high surgical risk. And again, it may be that the increase of aortic valve replacements in both surgical and nonsurgical groups, may be due to the inclusion of high-risk patients who weren't previously evaluated before TAVR program initiation. TAVR volume did exceed SAVR volume in 5 out of 7 hospitals' 4-year TAVR programs, and obviously, until recently, TAVR has been reserved for high-risk patients; however, recent studies have shown similar results in terms of mortality and morbidity in high-risk patients. The influence of TAVR on surgical volumes in California has obviously shown an overall steady balance in terms of the overall volume, but at the individual hospitals the volume has significantly changed in hospitals with 4-year programs. Obviously, needs to be more data on these patients in terms of their long-term outcomes, in terms of the long-term viability of the aortic valve, of the TAVRs compared with SAVRs, and the characteristics of these patients. What kind of patients are undergoing these procedures? Is it high-risk patients, intermediate-risk patients, and low-risk patients, or is it just high-risk and intermediate-risk patients? These are the questions we hope to further analyze and get the answers to in the future.

Dr Weyant. The discussion will be opened by Dr Joseph Woo from Stanford.

Dr Woo. I'd like to thank the Association for the opportunity to discuss. I congratulate the authors on a wellresearched and presented analysis of California state data on the influence of TAVR program implementation on SAVR volumes. From the data, the authors conclude that after implementation of the TAVR program, the SAVR volume initially increases, plateaus, then decreases over time 
as TAVR volume increases. Regardless of the causes of this volume shift, which we'll discuss later, I congratulate the authors on their efforts, which reinforce the importance of active surgeon involvement in TAVR decision making, procedural performance, and research.

I have 3 questions. The first is, we postulate that the changes in volume are due to a shift in SAVR candidates to TAVR without specific individual clinical data and referral information, which we cannot obtain from the administrative dataset, as you commented. This is rather difficult to prove. Might you comment on potential other hypotheses such as that the SAVR pool of candidates and the TAVR pool of candidates are somewhat independent and that over time, what's happening is that there are really changes in what are viewed as potential candidates for surgical AVR and transcatheter AVR, because both of these capabilities and technologies change.

Dr Maximus. Yeah. I agree. I think that the top cause for the increase we see in overall volume is the referral patterns of maybe practitioners, cardiologists, people who probably weren't being referred because it was believed that they were probably too sick to undergo a surgical procedure and probably the practitioner is saying, I can refer this guy for a minimally invasive procedure and it turns out now that they're fully evaluated is that they can undergo a surgical procedure, so I think that's probably the reason why you see that initial increase in the pool of candidates for either TAVR and SAVR.

Dr Weyant. So on a more general level, as the early ex vivo pulse duplicator data showed and more recently, as recently as last month at EuroPCR, there is an early signal that there may be advanced or accelerated structural valve deterioration among TAVRs.

Dr Maximus. Correct.

Dr Weyant. If this early signal progresses and, in fact, TAVRs are more prone to early deterioration, might there be a shift in volume back toward SAVR?

Dr Maximus. Absolutely, and that's a good point that you bring up. An abstract actually from just a month ago where they did a Kaplan-Meier analysis and they thought that possibly there was up to a 50\% degeneration of the TAVR valves. I think that's an excellent point. I think in a high-risk patient, TAVR has already been shown to be a great alternative to medical management. However, a low-risk patient, you know, the 40-year-old who needs a valve replacement, is a lot different from the 85-year-old who needs a valve replacement. So we don't know how long these valves are going to last, there just aren't enough data. So I think ultimately, depending on what the long-term data show, that's going to have a huge influence on these volumes and these referral patterns, ultimately.

Dr Weyant. So as a correlate to your answer, do low-risk trials make sense at this point?
Dr Maximus. I don't think they make sense at this point. I think you need more long-term data.

Dr Weyant. So then along the lines of examining statewide data, the Virginia Cardiac Surgery Quality Initiative presented, or reported this year, that TAVR actually costs almost double SAVR, and that this cost differential was most pronounced in the intermediate-risk groups, so because we're all talking about valve and TAVR is so much more expensive, particularly in the less risky groups, does it make sense that perhaps at some point, SAVR actually becomes the value proposition and again shifts volume?

Dr Maximus. Absolutely. I think that's very possible. There's actually another study, too, that came out that used data from the Observational Study of Effectiveness of SAVR-TAVI Procedures for Severe Aortic Stenosis Treatment (OBSERVANT) trial and they actually showed that TAVR...excuse me, that SAVR was superior in the low-risk patient population, that was only based on, again, 3-year survival data so it was limited, but again, in terms of cost, absolutely. If in these low-risk and intermediaterisk patients it's a cost benefit and we don't know the long-term outcomes, then I think surgical replacement is, at this point, obviously a more viable option until we have more data.

Dr Weyant. I congratulate you on a very balanced view of the field.

Dr Maximus. Thank you.

Dr Weyant. Over here, Dr Conte?

Dr Conte. I would like to congratulate you as well on a nice presentation of a very important topic.

Dr Maximus. Thank you.

Dr Conte. I'd like to reiterate what Dr Woo suggested. I think it's imperative that surgeons are very, very involved, not only from the standpoint of doing the procedures, but identifying the patients that truly benefit from TAVR, that there are certainly patients who would benefit from surgery and we have to be there because clearly, our experience in treating aortic stenosis is far greater and our insights are far greater than those of our cardiology colleagues. On the other hand, I actually take some opposition to Dr Woo because I don't think there's an economic argument. Because our patients are going directly from the cath lab to the floor and going home on postoperative day 1, and the costs of valves are going down exponentially, we pay less now, $50 \%$ less now for a valve clinically than we did during any of the trials. There will be an economic advantage to TAVR, so I don't think that trying to make that argument will carry much weight. But the question I have for you is what have you done at UCLA, I know this is statewide data, but just for perspective's sake, what have you guys done at UCLA to include residents trained in the TAVR program, because this is going to be a part of what we as surgeons do going forward, and what have you done in cardiac surgery practice to make cardiac surgery 
more inviting; that is, have you done more hybrid procedures, have you done more minimally invasive procedures? Do you think that by making surgery more attractive from that standpoint, no blood transfusions, discharge on day 3, we will perhaps accentuate what I think will be durability advantages to surgery for a variety of reasons on a much quicker time scale?

Dr Maximus. I appreciate the questions, Dr Conte. I have to correct you, I'm actually at UC Irvine, not UCLA, Dr Shemin from UCLA was among the coresearchers, but at UC Irvine, we don't have a TAVR program, yet I know they're in the process of developing that, to answer your question in terms of that. I have been very involved with Dr Milliken who's the cardiothoracic surgeon at UC Irvine and it has been a joint project in terms of developing this program, getting insight from both cardiothoracic surgery and cardiology for the reasons you mention in terms of the cardiothoracic surgeons should be involved in determining which patients are going to be involved for TAVR. In terms of resident exposure to TAVRs and these types of procedures, being a resident, a fifth-year surgery resident, I would say that is, you know, the new technology is something that is interesting to younger surgeons, I mean, that's among the things that attracts me to the different, attracted me to surgery initially, was the evolving technology and I think that is something that should be considered in terms of training, you know, wire skills and things like that for cardiothoracic surgery training in the future, because I think this is something that is going to be involved, whether we like it or not.

Dr Cleveland. Enjoyed your presentation.

Dr Maximus. Thank you.
Dr Cleveland. My question has to do with how you can, again, maybe get a more granular look at these data or not. At least 2 of the hospitals, I'm guessing, were large contributors to intermediate-risk trials that may have, if you will, different groups of patients. So if you look at those, can you figure out if it's possible that 1 of those patients who had access to the intermediate trial, because we saw these patients creep into the TAVR world. It is a possibility for bias and perhaps who's getting SAVR versus TAVR, we'll be able to look back.

Dr Maximus. Absolutely. We didn't have access to which hospital was involved with the intermediate trialsthat's something we hope to gain access to. The other thing we hope to gain or we're working on getting is actually the actual cost analysis to see how much these procedures cost for each hospital and what the total cost was as well.

Dr Weyant. Dr Shemin, quick comment, please.

Dr Shemin. I just want to follow-up on John Conte's question. There is no doubt that cardiac surgeons need to think of themselves as interventional cardiac surgeons. Our specialty is going across the interventional world as well as the open surgical world. Training programs need to expose every resident to the heart team concept, how to do joint evaluation, make decisions on different technologies, and what's best for the patient evidence-based and as the former chair of the board, Cam Wright, is here and Dave Fullerton, the current director, I think the American Board of Thoracic Surgery, when they start requiring the case list, interventional procedures, and minimally invasive cardiac surgery, we will finally have every training program and every trainee actually getting the experience and indeed be able to work in the current system in the future.

Dr Weyant. Thank you. 